\title{
Handover Control Function Based Handover for Mobile IPv6
}

\author{
Guozhi Wei ${ }^{1}$, Anne Wei ${ }^{1}, \mathrm{Ke} \mathrm{Xu}^{2}$, and Hui Deng ${ }^{3}$ \\ ${ }^{1}$ Université de Paris XII 122 Rue Paul, \\ Armangot 94400 Vitry-sur-Seine, France \\ \{Guozhi.Wei, Wei\} @univ-paris12.fr \\ ${ }^{2}$ Department of Computer Science and Technology, \\ Tsinghua University, China \\ Xukeacsnet1.cs.tsinghua.edu.cn \\ ${ }^{3}$ Hitachi (China) Investment, Ltd, Beijing, China \\ Hdeng@hitachi.cn
}

\begin{abstract}
IEEE 802.11 Wireless LAN (WLAN) has been enthusiastically adopted in business offices, homes, and other spaces, for both public and private wireless local network connection. The users would like to deploy Voice IP (VoIP) and Video Phone based on Mobile IPv6 Protocol over Wireless LAN network. However, the frequent change in the mobile node's location causes evident signaling overhead, handover latency and packet loss, which in turn leads to the service degradation of real time traffic in Mobile IPv6. In this paper, we propose a scheme based on Wireless LAN by adding a new component called Handover Control Function (HCF) in MIPv6, which records all APs's MAC address, backend ARs' address and network prefix of those AP's. By the means that all Mobile Nodes (MNs) report periodically all AP's MAC address and signal strength information to HCF which MN can probe, HCF decides whether or which AP MN shall associate with and notifies $\mathrm{MN}$ about the new AP/AR's information, meanwhile, a bi-cast mechanism shall be applied to further improve handover performance by reducing the packet loss during handover.
\end{abstract}

\section{Introduction}

Wireless LAN (WLAN) technologies, especially the IEEE 802.11 standards [1], have got great attention in recent years. A growing number of WLANs have been set up in public buildings or corporate environments as access networks to the Internet. These WLANs are connected to the Internet through layer 2 Access Points (APs) and layer 3 Access Routers (ARs). In WLAN, users could freely change their places when they are communicating with other users. However the real-time applications (such as VoIP and Video Phone) couldn't be supported due to long handover delays and high packet losses brought by the handover process. The handover process occurs when MNs moves from one AP/AR to another.

In order to support the mobility of MNs, Mobile IPv6 (MIPv6) [2] is proposed by the Internet Engineering Task Force (IETF), which describes the protocol operations 
for $\mathrm{MN}$ to maintain connectivity with the Internet as it moves between subnets. These operations involve movement detection, IP address configuration, and location update. Many approaches are submitted to improve the performance of handover and to meet the requirements of real-time applications.

In Consideration of Wireless LAN's special feature, we propound a scheme to achieve MIPv6 fast handover in WLAN by introducing a new component called Handover Control Function (HCF) in Hierarchical Mobile IPv6 (HMIPv6) [3].

The remainder of the paper is organized as follows. Section 2 gives a brief resume to previous works related to handover management in Mobile IPv6. Section 3 presents our HCF Based Handover for MIPv6 scheme and the detailed protocol operation. Analysis and comparison are shown in section 4. Finally, conclusion and future works are mentioned in section 5 .

\section{Background and Related Works}

Recently, several extensions to MIPv6 have been proposed aimed to reduce the handover latency and packet loss.

Actually, the main proposals accepted by IETF are Hierarchical Mobile IPv6 (HMIPv6) and Fast Handover for MIPv6 (FHMIPv6). HMIPv6 [3, 4] introduces Mobility Anchor Point (MAP) (a special node located in the network visited by MN) who acts somewhat like a local Home Agent (HA) for the visiting MN. Moreover, HMIPv6 separates MN mobility into micro-mobility (within one domain or within the same MAP) and macro-mobility (between domains or between MAPs). With this hierarchical network structure, MAP can limit the amount of signaling required outside the MAP's domain. Therefore, the amount and latency of signaling between a $\mathrm{MN}$, its HA and one or more Correspondence Nodes (CNs) decrease. Consequently, the layer 3 handover delays are reduced.

FHMIPv6 [5] reduces packets loss by providing fast IP connectivity as soon as a new link is established. The network uses layer 2 triggers to launch either PreRegistration or Post-Registration handover scheme [6]. In Pre-Registration scheme, the network provides support for preconfiguration of link information (such as the subnet prefix) in the new subnet while MN is still attached to the old subnet. By reducing the preconfiguration time on the new subnet, it enables IP connectivity to be restored at the new point of attachment sooner than would otherwise be possible. In Post-Registration scheme, by tunneling data between the previous AP/AR and new AP/AR, the packets delivered to the old Care of Address (CoA) are forwarded to the new CoA during link configuration and binding update. So it is possible to provide IP connectivity in advance of actual Mobile IP registration with the HA or CN.

Besides the main proposals, there have been numerous approaches for providing lossless handover and minimizing the handover delay. H.Chaouchi [7] propounded a Pre-Handover Signaling (PHS) protocol to support the triggering of a predictive handover and to allow the network to achieve accurate handover decisions 
considering different constraints such as QoS, the user profile and the mobile node service requirements. Y.Bi [8] submitted a Hierarchical Network-layer Mobility Management (HNMM) framework in which an integrated IP-layer handover solution is proposed to provide optimized network connectivity. The solution includes Enhanced IP-layer Handover mechanism (EIHO) with combined policies and Adaptive IP-layer Handover Control scheme (AIHC). Based on the IPv6 Soft Handover mechanism proposed by E.Perkins [9], the Competition based Soft Handover Management (CSHM) protocol of J. Kristiansson [10], the Multi-path Transmission Algorithm of S. Kashihara [11] are also proposed to decrease packet loss during handover.

\section{Handover Control Function Based Handover for Mobile IPv6}

In our paper, we focus on optimizing handover performance in MIPv6 over WLAN. When being used as link layer, WLAN doesn't permit users to employ FHMIPv6. Indeed, in WLAN, when MN moves towards a new AP, it must disconnect from its previous AP and scan for others APs in range. Based on the result of these scans, it will choose its new AP. Hence, there is no possibility for MN to use neither Preregistration nor Post-registration mechanism as MN doesn't know in advance where to go. Furthermore, once a new AP is selected, MN is no longer connected with its previous AP.

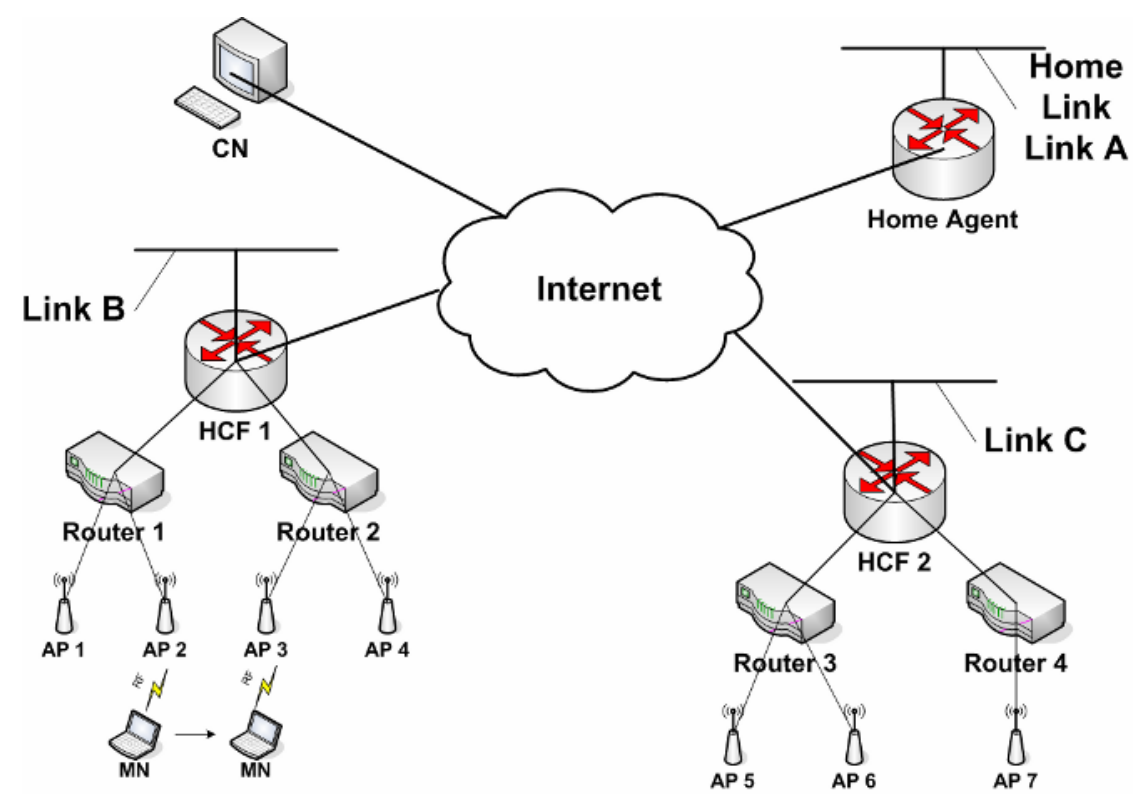

Fig. 1. HCF Based Handover for Mobile IPv6 
To resolve this problem, IEEE 802.11f standard, known as Inter-Access Point Protocol (IAPP) [12] [13] has been proposed. IAPP enables the WLAN's APs to communicate with each other. By using IAPP, FHIMPv6 could be applied in WLAN. However, IAPP can neither reduce greatly the layer 2 delay nor avoid the packet losses. Furthermore, it couldn't provide the global management, such as dynamic load balancing on APs and ARs.

Therefore, we introduce a new component Handover Control Function (HCF) and add two new messages --- HCFReq and HCFRep messages in Mobile IPv6 in order to resolve issues mentioned above. The architecture of the HCF Based Handover for Mobile IPv6 is shown in figure 1.

In this network scenario, one AR might connect to multiple APs. Those APs might have same network prefix or different network prefix. While MN moves from AP2 to AP3, the attached Access Router also changes from AR1 to AR2 as well.

MN reports periodically to HCF all APs' MAC addresses and signal strengths that MN can probe. Based upon those reported information such as AP's loading and MN's movement, etc, by using a predefined algorithm, HCF decides whether or which AP MN shall associate with and notifies MN about the new AP/AR's information, such as AP's MAC address, AR interface address, and network prefix. HCF decides which AR's interface MN should move to as well. Consequently, the new network prefix of MN will be notified by HCF through HCFRep message accordingly.

The "IPv6 address allocation and assignment policy" draft issued by RIPE NCC [14] provides the guidelines for allocation and distribution of IPv6 addresses. This draft reveals that in an IPv6 access network as MN moves across the subnets, the only change in its IPv6 address occurs in subnet identifier field of the address. The remaining portion of the address, including 48 bit global routing prefix and the 64 bit interface identifier remains unchanged. Moreover, in our proposal, MN's interface identifier is allocated according to the norm of EUI-64. It ensures that the MN's new CoA is unique in Mobile IPv6. Consequently, MN could configure its new CoA and begin to launch the binding update process even if it is still attached with previous AP/AR. HCF also knows MN's new CoA according to MN's old CoA and MN's new network prefix. Furthermore, Duplicated Address Detection (DAD) can be omitted during handover.

In [15], a pure IPv6 Soft handover mechanism is presented. It provides data transmission continuity for delay constrained applications. Respiring from this method, a bi-casting mechanism was proposed and was applied to further improve handover performance. HCF acts as an extension of MAP in HMIPv6 which could begin to bicast traffic to both MN's old CoA and new CoA after sending HCFRep to $\mathrm{MN}$ to reduce packet loss during handover. HCF Bicast traffic also removes the timing ambiguity, regarding when to start sending traffic to MN's new point of attachment following a Fast Handover. It also minimizes service disruption time in the case of ping-pong movement.

Figure 2 shows messages exchange in the handover procedure. 


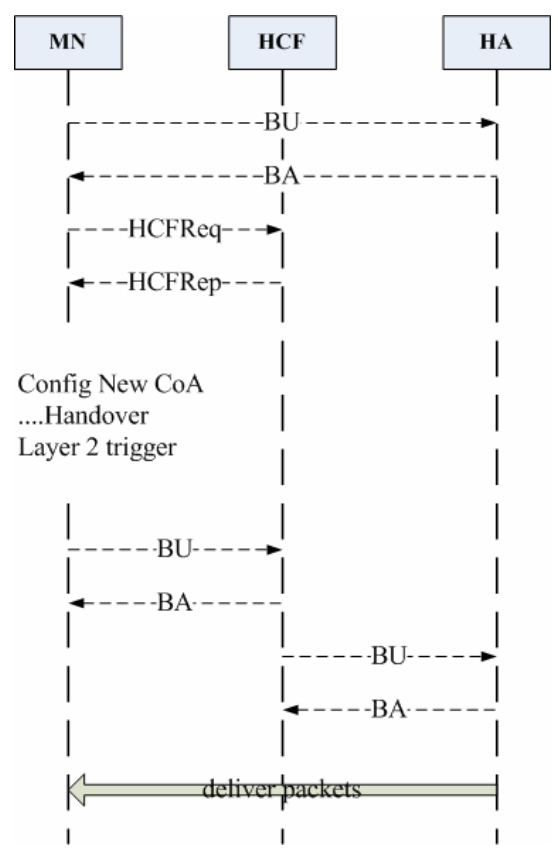

Fig. 2. Protocol for HCF based handover

1. When MN registers to HA at the first time, it will send Binding Update message to HA, and HA will response with Binding Acknowledgement.

2. MN probes all neighbor AP's information, including signal strength. Once the signal strength threshold is gotten over, MN shall send HCFReq message directly to HCF to report the information of its neighbor AP.

3. After HCF receives the HCFReq message, it will decide whether or which AR/AP $\mathrm{MN}$ shall associate with.

4. Detail algorithm for HCF judgment of MN handover mostly is based mostly on the signal strength that MN received from neighbor APs and HCF's network administrating policy.

5. HCF judge $\mathrm{MN}$ in some sense that can also help loading balance among different ARs and APs, if the number of registered MNs in one AR or AP have reached a limit, HCF will not approve MN to move to that network.

6. After HCF makes decision that which AR/AP MN shall move to, HCF will notify MN about new AR/AP's information, such as link prefix and AR's address. The information will help MN make a new CoA before it handover. This address also has been known by HCF since new CoA is made based on EUI-64.

7. After MN receive the HCFRep message, it knows that which AR/AP it will associate with and will configure its new CoA based on HCFRep message about new AP/AR.

8. When MN moves from AP2 to AP3, the attached Access Router also changes from AR1 to AR2 accordingly. MN will intensively disassociate with AP2 and associate with AP3. 
9. HCF works as an extension of MAP, and bicast traffic is sent out from HCF to both MN's old CoA and new CoA. Once MN attaches AP3/AR2, that traffic will go directly to MN's new CoA. After receiving the new binding update from new CoA, $\mathrm{HCF}$ will remove the traffic which goes to old CoA.

\section{Analyzing and Evaluating}

To better analyze handover delay, we divide the handover delay into two parts: layer 2 handover delay and layer 3 handover delay, seen in figure 3 . In the layer 2 , the delays include the time for movement detection, channel scan, authentication, detection and re-association. In the layer 3, the delays contain: (1) the time that MN detects the AR changing by receiving Router Advertisement (RA) from new AR. (2) The time that MN negotiates with new AR to get access admission. It includes $\mathrm{MN}$ authentication, CoA configuration and related resources configurations in AR. (3) the time for Mobile IP registration. It includes the time that $\mathrm{MN}$ sends Binding Update to $\mathrm{CN}, \mathrm{HA}$ and receives BU ACK.

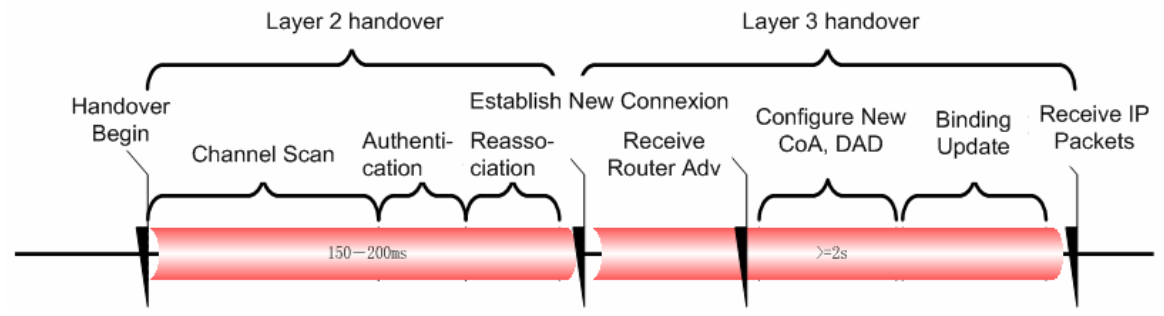

Fig. 3. Layer 2 and Layer 3 Handover Process

As shown in figure 3, the handover delay is too high to meet a real-time application need. We try to reduce the processing time or to omit unnecessary process in our approach. Compared with other proposals, HCF decides whether or which AP MN shall attach to before MN disconnects with its previous AP. By this means, MN reduces the time of channel scanning and switching phase running - authentication and re-association requests - with new AP. Being notified of the new network prefix by $\mathrm{HCF}, \mathrm{MN}$ is informed of the new AR/AP's information, and gets its new CoA. So that it doesn't need either to wait for Router Advertisement message or to execute the new CoA configuration process. Besides, since unique IP address is no longer necessary to be verified again, DAD process execution can be omitted accordingly. Moreover, Pre-registration mechanism is permitted to be used in our proposal, hence, $\mathrm{MN}$ could carrying on binding update with its $\mathrm{HA}$ and $\mathrm{CNs}$ before leaving its previous AP. In conclusion, handover delay could be greatly minimized, and our proposal will be proved by the future simulation.

Packet Loss Rate (PLR) is another element which influences the handover performance. Packet Loss Rate is influenced not only by L3 handover delay, but also by L2 handover delay. The low packet loss rate can be realized by minimizing the 
handover delays. As we mentioned that our scheme could cut down either the layer 2 or layer 3 handover delay. In addition to decrease the handover delays, there are some other means to reduce the packet loss. Some proposals want to reduce the packet loss by buffering packets in old AP/AR when L2 handover is detected and forwarding packets to new AP/AR as soon as L2 handover ends. While this increases the charge of AP/AR and the signaling exchange in the system. Some proposals reduce the packet loss by using the IPv6 Soft handover mechanism, which sends the traffic to both MN's old CoA and new CoA while it needs the precise time control. In our scheme, HCF decides which AR/AP MN shall attach to, and HCF decides exactly how and when to send and end bicast traffic to which AR/AP.

\section{Conclusion and Prospects}

In this paper, we propose a simple but effective scheme, which could reduce the handover latency and packet losses without modifying AP/AR of WLAN. Our proposal permits $\mathrm{MN}$ to get the new $\mathrm{CoA}$ and to lance binding update process before moving to the new AR/AP. Moreover, the omission of DAD process optimizes greatly handover performance. Furthermore, by the means of sending bicast traffic from HCF to both old and new CoA, the packet loss could be minimized.

This paper expounds our primary concept. In the next step, our proposal will be simulated and evaluated by using OPNET (a discrete network simulation tool) [16]. In our future study, we are about to deal with the other MIPv6 issues, such as AAA, QoS and to enable $\mathrm{HCF}$ to better manage the mobility and the network resource in the WLAN.

\section{References}

1. "IEEE 802.11b: Wireless LAN Medium Access Control (MAC) and Physical Layer (PHY) Specifications," IEEE Standard, 1999.

2. D.Johnson, C.Perkins, and J.Arkko, "Mobility Support in IPv6", RFC 3775, June 2004.

3. H.Soliman, C.Castelluccia, K.Malki, and L.Bellier, "Hierarchical Mobile IPv6 mobility management (HMIPv6)", RFC 4140, August 2005.

4. Wei Kuang Lai, Jung Chia Chiu, "Improving Handoff Performance in Wireless Overlay Networks by Switching Between Two-Layer IPv6 and One-Layer IPv6 Addressing," IEEE JOURNAL ON SELECTED AREAS IN COMMUNICATIONS, VOL. 23, NO. 11, NOVEMBER 2005.

5. R. Koodli, Ed."Fast Handovers for Mobile IPv6", RFC 4068, July 2005.

6. C.Blondia, O.Casals, et al. "Performance Evaluation of Layer 3 Low Latency Handoff Mechanisms," Mobile Network and Application 9, pp.633-645, 2004

7. H.Chaouchi, P.Antunes, "Pre-handover signaling for QoS aware mobility management," INTERNATIONAL JOURNAL OF NETWORK MANAGEMENT 14, pp.367-374, 2004;

8. Y.Bi, P.Iyer et al. "An Integrated IP-layer Handover Solution for Next Generation IPbased Wireless Network", Vehicular Technology Conference, 2004 Vol. 6 pp.3950 - 3954

9. E.Perkins, "IP mobility support for IPv4", RFC 3220, January 2002.

10. J. Kristiansson and P. Parnes, "Application-layer Mobility support for Streaming Realtime Media," Wireless Communications and Networking Conference, Vol.1 pp.268-273, 2004. 
11. S. Kashihara, K. Iida, H. Koga, Y. Kadobayashi, and S. Yamaguchi, "End-to-End Seamless Handover using Multi-path Transmission Algorithm," In Internet Conference 2002

12. "IEEE 802.11f: Recommended Practice for Multi-Vender Access Point Interoperability via an Inter-Access Point Protocol Access Distribution Systems Supporting IEEE 802.11 Operation,” IEEE Standard 802.11f/D1, Jan. 2002 (draft).

13. Chun-Ting Chou and Kang G. Shin, "An Enhanced Inter-Access Point Protocol for Uniform Intra and Intersubnet Handoffs," IEEE TRANSACTIONS ON MOBILE COMPUTING, VOL. 4, NO. 4, JULY/AUGUST 2005

14. IPv6 Address Allocation and Assignment Policy, ripe-267, January 2003. http://www.ripe.net/ripe/docs/ipv6policy.html

15. F.Belghoul, Y.Moret, C.Bonnet, "IP-Based Handover Management over Heterogeneous Wireless Networks", Local Computer Networks, 2003. LCN '03. Proceedings. 28th Annual IEEE International Conference on 20-24 Oct. 2003 pp.772 - 773

16. www.opnet.com 